\title{
BLIND MULTIUSER IDENTIFICATION AND DETECTION IN CDMA SYSTEMS
}

\author{
Javier R. Fonollosa†, José A. R. Fonollosał, Zoran Zvonarł and Josep Vidal† \\ †Universitat Politècnica de Catalunya \\ Department of Signal Theory and Communications \\ 08034 Barcelona, SPAIN \\ fono@tsc.upc.es \\ †Analog Devices \\ Communications Division \\ Wilmington MA 01887-1024, USA \\ Zoran.Zvonar@analog.com
}

\begin{abstract}
Multiuser detection in Code Division Multiple Access systems usually requires either knowledge of the transmitted signature sequences and channel state information or use of a known training sequence for adaptation. In this paper we develop a scheme that can be employed for the joint adaptive blind multiuser identification and detection in asynchronous CDMA systems. This scheme relies on a multiuser Viterbi algorithm that incorporates an adaptive estimation of the overall channel impulse responses, given by the convolution of the signature sequences of the users and corresponding physical channels impulse responses. Once the overall channel responses are estimated, the blind multiuser detection algorithm performs like the maximumlikelihood sequence estimator. Results are provided to illustrate the convergence of the blind multiuser approach, near-far resistance and sensitivity to the algorithm initialization.
\end{abstract}

\section{INTRODUCTION}

Increasing interest in the multiuser detection area was initiated by the work on the optimal structures and the performance bounds for the Gaussian noise channel [1]. The optimal multiuser detector employs a bank of matched filters (one for each active user) followed by a multiuser maximum-likelihood sequence estimator (MLSE). Its complexity is exponential in the number of active users and it requires knowledge of the transmitted signature sequences and channel state information. Consequently, suboptimal near-far resistant receivers of linear complexity were developed that still provide significant performance gain over the conventional single user receiver [2]. In addition, multiuser receivers were designed for fading and multipath channels [3].

Recently, adaptive multiuser receivers have gained considerable attention [4]. The requirement of train-

This work was supported by CICYT of Spain, Grant number TIC92-0800-C05-05. ing sequences for adaptive multiuser reception, or previous knowledge of the users signatures and channels for the centralized multiuser receivers mentioned above, represents an important drawback for efficient multiuser communications. This motivates the development of multiuser receiver structures that can be adapted blindly, i. e. without the need of training sequences [4]. In this paper we present a scheme for the joint blind identification and detection of multiuser signals which has the following features:

- No knowledge of the signature waveform of any user is required (although their approximate knowledge speeds up the convergence).

- Training sequences are not required.

- No timing information of any user needs to be known or acquired a priori.

- No channel state information is required.

- The blind multiuser detector is near-far resistant.

- It provides an estimate of the signature sequence of each user convolved with its physical channel impulse response, and its ML transmitted sequence estimate after the convergence.

This approach is motivated by the bandwidth efficient multiuser systems which utilize small spreading ratio, burst transmissions and relatively large SNR [5].

\section{SYSTEM MODEL}

We consider the asynchronous CDMA white Gaussian noise channel,

$$
r(t)=\sum_{n} \sum_{k=1}^{K} b_{k}[n] h_{k}(t-n T, t)+\sigma w(t)
$$

where $h_{k}(t-n T, t)$ is the overall complex channel impulse response, given by the convolution of the signature sequence, physical channel and the receiving filter 
impulse responses. It incorporates the amplitude and the delay for user $k$, and its duration is assumed to be smaller or equal to $L$ symbols, i.e. $h_{k}(\tau, t)=0, \tau<$ $0, \tau>L T, \forall t$. The total number of active users is $K$ and their transmitted sequences are binary independent symbols $b_{k}[n] \in\{1,-1\}$. The symbol rate is $1 / T$ and $w(t)$ is normalized white Gaussian noise.

The CDMA channel is sampled at a rate $M / T=$ $1 / T_{s}$ to derive the discrete vector sequence $\mathbf{r}[n]$

$$
\mathbf{r}[n]=\left[r(n T), r\left(n T+T_{s}\right), \ldots, r\left(n T+(M-1) T_{s}\right)\right]^{T} .
$$

The observation $\mathbf{r}[n]$ is modeled as a probabilistic $M$ length vector sequence of a state vector $s[n]$

$$
\mathbf{r}[n]=\mathcal{H}[n] \mathbf{s}[n]+\mathbf{w}[n] .
$$

There are $N=2^{L K}$ possible state vectors corresponding to $L$ binary symbols of $K$ users. We denote the individual states as the $K L$ length vectors $\left\{\mathbf{s}_{1}, \mathbf{s}_{2}, \ldots, \mathbf{s}_{N}\right\}$, $\mathrm{s}_{i}=\left[A_{i}(1,1), \ldots, A_{i}(L, 1), \ldots, A_{i}(L, K)\right]^{T}, A_{i}(l, k) \in$ $\{1,-1\}$ and the state at time instant $n T$ as $\mathbf{s}[n]$.The $(M \times K L)$ matrix $\mathcal{H}[n]$ depends of the overall discrete impulse response for all users

$$
\begin{gathered}
\mathcal{H}[n]=\left[\mathbf{H}_{1}[n], \ldots, \mathbf{H}_{K}[n]\right] \\
\mathbf{H}_{k}[n]=\left[\mathbf{h}_{k 0}[n], \ldots, \mathbf{h}_{k(L-1)}[n]\right] \\
h_{k}((n+l) T, n T) \\
\vdots \\
{\left[\begin{array}{c} 
\\
h_{k}\left((n+l) T+(M-1) T_{s}, n T\right)
\end{array}\right]}
\end{gathered}
$$

The normalized noise vector is defined as

$$
\mathbf{w}[n]=\left[w(n T), w\left(n T+T_{s}\right), \ldots, w\left(n T+(M-1) T_{s}\right)\right]^{T}
$$

The probability density function of the observation vector conditioned on a given individual state $s_{j}$ is

$$
p\left(\mathbf{r}[n] \mid \mathbf{s}_{j}\right)=\frac{1}{\sigma \sqrt{2 \pi}} \exp \left(-\frac{\left(\mathbf{r}-\mathbf{m}_{j}\right)^{H}\left(\mathbf{r}-\mathbf{m}_{j}\right)}{2 \sigma^{2}}\right)
$$

where

$$
\mathbf{m}_{j}=\mathcal{H}(n T) \mathbf{s}_{j}, \quad 1 \leq j \leq N
$$

\section{BLIND IDENTIFICATION AND DETECTION ALGORITHM}

\subsection{Introduction}

If the overall impulse response for each user was known, that is if the signature sequence, physical channel impulse response, amplitude and delay corresponding to each user were available, then using this information, the Viterbi algorithm could be employed to determine the multiuser maximum-likelihood sequence. That is, given $\mathcal{H}(t)$, and all possible multiuser sequences $\mathbf{b}=\left\{\left[b_{1}[n], \ldots, b_{K}[n]\right]^{T^{T}}, \forall n\right\}$ the $\mathrm{ML}$ transmitted sequence is the one that maximizes the joint posterior distribution $p(\mathbf{b} \mid r(t), \forall t)$. This maximization, equivalent for equiprobable sequences to $p(r(t), \forall t \mid \mathbf{b})$, can be efficiently computed finding the most likely sequence of states using (8) for cumulative metric calculation and the Viterbi algorithm [1].

\subsection{Algorithm description}

In the method we present, the Viterbi algorithm is applied with current estimates of the overall impulse responses which are updated recursively. First the algorithm is initialized with an arbitrary impulse responses for each user. The number of users $K$ is assumed known together with a bound for the impulse response duration that determines $L$. Then the algorithm is iterated at the symbol rate as in a conventional Viterbi scheme. In this case however, metrics along the trellis are computed using for each state its own current estimate of the overall impulse response, $\hat{\mathcal{H}}_{j}[n], j=1, \ldots, N$. At each iteration, a $\mathrm{ML}$ path is continued for each state selecting the most likely possible predecessor and updating the accumulated metric using (8). Each state shares $L-1$ symbols for each user with its set of possible predecessors and thus there are $2^{K}$ predecessors per state.

The channel estimate matrix $\hat{\mathcal{H}}_{j}[n], j=1, \ldots, N$ is updated for each state modifying the one associated to its most likely predecessor using a standard system identification LMS scheme

$$
\hat{\mathcal{H}}_{j}[n]=\hat{\mathcal{H}}_{i}[n-1]+\mu \mathbf{e}_{i j}^{*}[n] \mathbf{s}_{j}^{T}
$$

where the state $\mathbf{s}_{i}$ is the predecessor to state $\mathbf{s}_{j}, \mu$ is the adaptation constant and the error vector $\mathbf{e}_{i j}[n]$ is defined as

$$
\mathbf{e}_{i j}[n]=\mathbf{r}[n]-\hat{\mathcal{H}}_{i}[n-1] \mathbf{s}_{j}
$$

After convergence, the impulse response corresponding to the most likely final state can be employed to yield the ML transmitted sequence for each user as in the conventional multiuser optimal receiver. A similar approach was proposed for the blind equalization of single user channels using the Viterbi algorithm [6] and the Baum-Welch identification algorithm [7].

\subsection{Algorithm convergence}

We illustrate the convergence of the algorithm in a scenario of 4 users employing Gold sequences of length 7 in stationary AWGN channel. Users' signals are synchronous and their amplitudes are 1, 1.5, 2, and 2.5 respectively. After 300 iterations (symbols) the global 


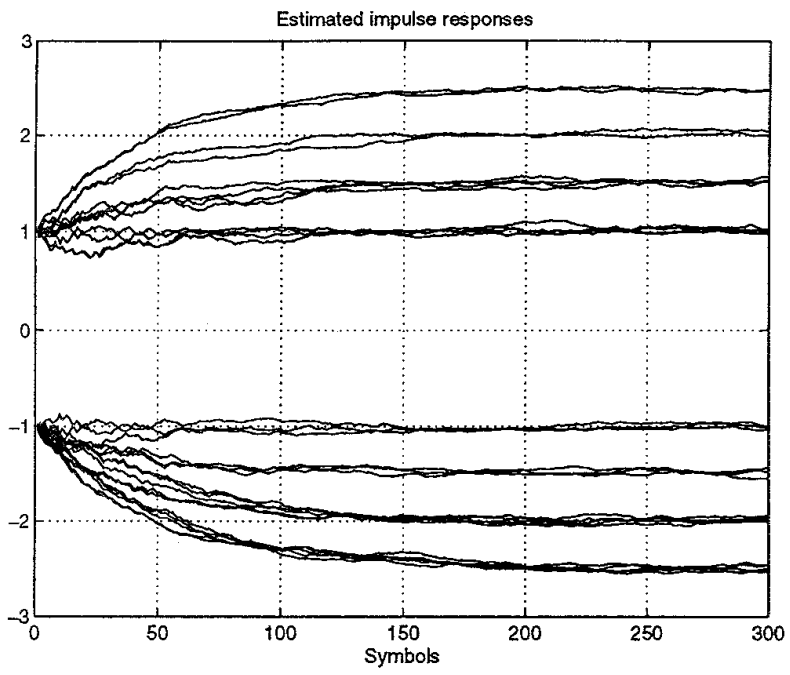

Figure 1: Learning curve for $\hat{\mathcal{H}}_{M L}$

estimated impulse response is

$$
\hat{\mathcal{H}}_{M L}=\left[\begin{array}{rrrr}
1.020 & 1.520 & -1.966 & -2.511 \\
0.986 & -1.477 & -1.939 & -2.532 \\
-1.002 & 1.551 & -1.967 & 2.471 \\
-1.033 & -1.547 & 1.991 & -2.467 \\
0.989 & -1.433 & 2.038 & -2.532 \\
-1.001 & 1.523 & -2.005 & -2.467 \\
1.013 & 1.501 & -1.993 & 2.474
\end{array}\right]
$$

whereas the actual $\mathcal{H}$ is

$$
\mathcal{H}=\left[\begin{array}{rrrr}
1 & 1.5 & -2 & -2.5 \\
1 & -1.5 & -2 & -2.5 \\
-1 & 1.5 & -2 & 2.5 \\
-1 & -1.5 & 2 & -2.5 \\
1 & -1.5 & 2 & -2.5 \\
-1 & 1.5 & -2 & -2.5 \\
1 & 1.5 & -2 & 2.5
\end{array}\right]
$$

After convergence, the final global impulse response could be used to yield a close to optimal demodulation of the received sequence. The algorithm was initialized assuming the amplitude of all users was 1 and the SNR was $10 \mathrm{~dB}$ for the weakest user. No symbol errors were observed backtracking the ML path created during adaptation. Fig. 1 illustrates the evolution of the estimation of $\mathcal{H}$ for the ML state at each sample.

\section{LOCAL MINIMA EFFECT}

\subsection{Existence of local minima}

In practice, this algorithm can be employed without further refinement if an approximate knowledge of the overall impulse response is used for initialization and all users are received with similar power. Otherwise, the adaptation can be easily trapped in local minima as a consequence of the near-far effect. This effect can be better understood in the two users case. When one of the users is characterized by an impulse response of much greater magnitude than the other, the algorithm tends to split the greater impulse response among both users (since this locally maximizes the probability function (8)) and the signal received from the weak user is just taken as part of the noise. The algorithm converges to a good approximation of the strong impulse response and neglects the weak one. The same effect can happen after random initialization of $\hat{\mathcal{H}}$. This phenomenon, however can be easily detected since the corresponding estimated $\mathrm{ML}$ sequences for both users are exactly the same (except for a possible sign change). In the more general case of $K$ users, any of their actual impulse responses, $\mathbf{H}_{k}[n]$ could in principle appear partitioned into an arbitrary number of users, i. e., $\mathbf{H}_{k}[n] \simeq \hat{\mathbf{H}}_{k}[n]+\sum_{j} \pm \hat{\mathbf{H}}_{k_{j}}[n]$, but still this effect will be reflected in identical estimated $\mathrm{ML}$ sequences for these users ( $k$ and $k_{j}$ ) again except for a possible sign difference.

\subsection{Avoiding local minima}

We propose a simple procedure to overcome the convergence to a local minima. Consider the two synchronous users case with $L=1$. An improved implementation of the algorithm checks every $P$ symbols for total coherence of the corresponding ML sequences for each user. The probability of random coincidence of two independent binary sources, except for a possible sign change, is $1 / 2^{P-1}$, and can be made arbitrarily small increasing $P$. In case this absolute coherence is detected, it is assumed that the algorithm has converged to a local minimum and the impulse responses of each user are added (or subtracted if the sequences were inverse) and assigned arbitrarily to one of them. The other user impulse response is just reset to zero, i. e.

$$
\begin{gathered}
\hat{\mathbf{h}}_{1}[n] \leftarrow \hat{\mathbf{h}}_{1}[n] \pm \hat{\mathbf{h}}_{2}[n] \\
\hat{\mathbf{h}}_{2}[n] \leftarrow \mathbf{0} .
\end{gathered}
$$

This compensation may be necessary until a good estimate of the strong impulse response is obtained which cancels the local minimum. In practice, we observed that one such compensations is necessary approximately for each $10 \mathrm{~dB}$ in power difference.

In general, for an arbitrary number of users and value of $L$, a total of $\left(\begin{array}{c}K \\ 2\end{array}\right)=K(K-1) / 2$ symbol crosscorrelation functions need to be computed, and each of them for $2 L-1$ lags. This cross-correlation functions are defined as

$$
\rho_{k k^{\prime}}[l]=\left\lfloor\frac{1}{P-|l|} \sum_{p=0}^{P-1} b_{k}[p+l] b_{k^{\prime}}[p]\right\rfloor ; \quad k^{\prime}>k
$$


where $b_{k}[p]$ is the ML sequence associated with user $k$ for the previous $P$ symbols. The global impulse response is compensated iterating for each user in ascending order, i.e. $k=1, \ldots, K$ the following impulse response update

$$
\hat{\mathbf{h}}_{k l}[n] \leftarrow \hat{\mathbf{h}}_{k l}[n]+\sum_{k^{\prime}=k+1}^{K} \sum_{l^{\prime}=0}^{L-1} \rho_{k k^{\prime}}\left[l-l^{\prime}\right] \hat{\mathbf{h}}_{k^{\prime} l^{\prime}}[n]
$$

and possible reinitialization,

$$
\begin{gathered}
\hat{\mathbf{h}}_{k^{\prime} l^{\prime}}[n] \leftarrow\left(1-\left|\rho_{k k^{\prime}}\left[l-l^{\prime}\right]\right|\right) \hat{\mathbf{h}}_{k^{\prime} l^{\prime}}[n] \\
k^{\prime}=k+1, \ldots, K ; l^{\prime}=0, \ldots, L-1
\end{gathered}
$$

\subsection{Near-far resistance}

We illustrate the near-far resistance of this blind deconvolution scheme considering the bandwidth efficient case [1] of 2 users using signatures $[1,1,1]$ and $[1,-1,1]$. The system is asynchronous and the second user arrives with a delay of $T_{s}$ with respect to the first one. This asynchronism makes $L=2$. Their amplitudes are 1000 and 1 respectively (60 dB power difference). After 400 iterations (symbols) and 6 coherence compensations, the global impulse response estimated for the ML sequences compared to the actual $\mathcal{H}$ is

$$
\begin{gathered}
\hat{\mathcal{H}}_{M L}=\left[\begin{array}{rrrr}
1000.0 & -0.0763 & -0.0199 & 1.0483 \\
1000.1 & 0.0385 & 0.9352 & 0.0361 \\
1000.0 & -0.0618 & -0.9724 & 0.0163
\end{array}\right] \\
\mathcal{H}=\left[\begin{array}{rrrr}
1000 & 0 & 0 & 1 \\
1000 & 0 & 1 & 0 \\
1000 & 0 & -1 & 0
\end{array}\right] .
\end{gathered}
$$

The number of symbol errors observed are 0 and 94 respectively (again backtracking the ML path created during adaptation and not recalculating the ML sequence with the estimated impulse responses). The algorithm was initialized assuming the amplitudes of all users were 1 and they were synchronous. The SNR was 64.7 and $4.7 \mathrm{~dB}$ respectively. Fig. 2 illustrates the evolution of the estimation of $\mathcal{H}$ for the ML state at each sample. The parameter $P$ was set to 30 . It can be observed how coherence compensations are necessary until a precise estimate of the strong impulse response is attained, approximately after 180 symbols. Afterwards, the algorithm converges to the global impulse response $\mathcal{H}$.

\section{CONCLUSIONS}

A new near-far resistant technique for the blind deconvolution of multiuser signals has been described. It can accommodate asynchronous multiuser channels with ISI with a complexity that is exponential in the

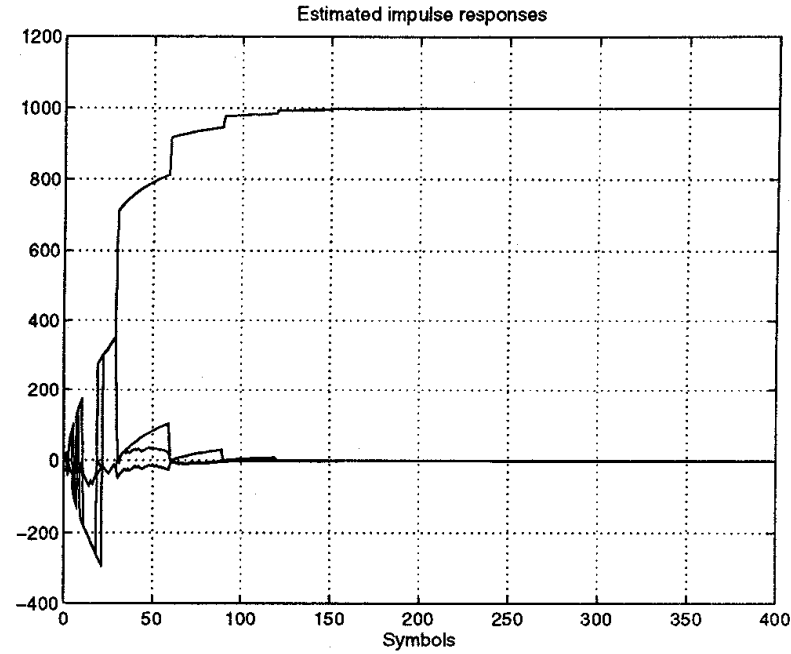

Figure 2: Learning curve for $\hat{\mathcal{H}}_{M L}$

number of users times the global impulse response duration in symbols. Local minima are avoided calculating cross-correlation functions between estimated ML sequences for each pair of users. The application of this method to fading and multichannel systems and experimental validation of the algorithm on the real data are the topics of further investigation.

\section{REFERENCES}

[1] Verdú, S., "Minimum Probability of Error for Asynchronous Gaussian Multiple-Access Channels," IEEE Trans. on Information Theory, Vol. IT-32, January 1986.

[2] Lupas, R. and Verdú, S., "Near-Far Resistance of Multiuser Detectors in Asynchronous Channels," IEEE Trans. on Communications, Vol. COM-38, March 1990.

[3] Zvonar, Z. and Brady, D., "Suboptimum Multiuser Detector for Synchronous CDMA FrequencySelective Rayleigh Fading Channels," IEEE Trans. on Communications, to appear.

[4] Verdú, S., "Adaptive Multiuser Detection," Proc. Third International Symposium on Spread Spectrum Techniques and Applications, Oulu, Finland, July 1994.

[5] Zvonar, Z., Brady, D. and Catipovic, J., "An Adaptive Linear Multiuser Receiver for Deep Water Acoustic Local Area Networks," Proc. IEEE ICASSP-94, April 1994.

[6] Seshadri, N., "Joint Data and Channel Estimation using Blind Trellis Search Techniques," IEEE Trans. on Communications, Vol COM-42, March 1994.

[7] Fonollosia, José A. R. and Vidal J., "Application of Hidden Markov Models to Blind Channel Characterization and Data Detection," Proc. IEEE ICASSP-94, April 1994. 